\title{
Effect of glucocorticoids on growth and bone mineral density in children with nephrotic syndrome
}

\author{
Diana Ribeiro • Sophie Zawadynski • Laure F. Pittet • \\ Thierry Chevalley • Eric Girardin • Paloma Parvex
}

Received: 19 August 2014 /Revised: 11 December 2014 / Accepted: 12 December 2014 / Published online: 10 January 2015

(C) Springer-Verlag Berlin Heidelberg 2015

\begin{abstract}
Glucocorticosteroids (GCs) are the first-line treatment for idiopathic nephrotic syndrome (NS), but prolonged administration interferes with growth and bone mineralization. We conducted a retrospective study to analyze the long-term impact of prednisone on growth and bone mineral density (BMD) in children with NS. Data from children with NS followed during almost 10 years were analyzed. Height and spine BMD values were converted to $Z$-scores (standard deviation $[\mathrm{SD}])$. The mean cumulative dose of GCs received was
\end{abstract}

Communicated by Jaan Toelen

D. Ribeiro · L. F. Pittet

Division of General Pediatrics, Department of Pediatrics, Children's

Hospital, University Hospitals of Geneva, 6 Rue Willy-Donzé,

1211 Geneva, Switzerland

D. Ribeiro

e-mail: ribeiro6@windowslive.com

L. F. Pittet

e-mail: Laure.Pittet@hcuge.ch

\section{S. Zawadynski}

Division of Nuclear Medicine and Molecular Imaging, Department of Imaging and Medical Information Sciences, University Hospitals of Geneva, 4 Rue Gabrielle-Perret-Gentil, 1211 Geneva, Switzerland e-mail: Sophie.Zawadynski@hcuge.ch

\section{T. Chevalley}

Division of Bone Diseases, Department of Medical Specialties, University Hospitals of Geneva, 4 Rue Gabrielle-Perret-Gentil, 1211 Geneva, Switzerland

e-mail: Thierry.Chevalley@hcuge.ch

E. Girardin $\cdot$ P. Parvex $(\varangle)$

Pediatric Nephrology Unit, Division of Pediatrics Specialties,

Department of Pediatrics, Children's Hospital, University Hospitals

of Geneva, 6 Rue Willy-Donzé 6, 1211 Geneva, Switzerland

e-mail: paloma.parvex@unige.ch

E. Girardin

e-mail: Eric.girardin@hcuge.ch calculated and correlated to patient's growth and spine BMD using linear regression and subgroup analysis. We included 30 patients diagnosed at 3.7 years old (interquartile range (IQR) 2.6-4.8) and followed over 9.8 years (IQR 6.6-11.7). The one half of NS patients was steroid sensitive and one half dependent or resistant. The median cumulative dose of GCs received was $0.27 \mathrm{mg} / \mathrm{kg} /$ day (IQR 0.18-0.35). Growth and spine BMD were both negatively associated with the cumulative dose of GCs $(P=0.001$ and $P=0.037$, respectively). Final height $Z$-scores were significantly lower in patients receiving $>0.2 \mathrm{mg} / \mathrm{kg} /$ day $\mathrm{GCs}(P=0.001)$. No difference was observed in spine BMD between subgroups.

Conclusion: Increasing doses of GCs were significantly associated with lower height and BMD Z-scores. A significant effect on growth was observed with cutoff doses above $0.2 \mathrm{mg} / \mathrm{kg} /$ day.

Keywords Glucocorticosteroids · Growth · Bone mineral density $\cdot$ Idiopathic nephrotic syndrome
Abbreviations
BMD Bone mineral density
BMI Body mass index
DXA Dual X-ray absorptiometry
GCs Glucocorticosteroids
IQR Interquartile range
NS Nephrotic syndrome
PQCT Peripheral quantitative computed tomography
SD Standard deviation
SDNS Steroid-dependent NS
SRNS Steroid-resistant NS
SSND Steroid-sensitive NS 


\section{Introduction}

Idiopathic nephrotic syndrome (NS) is the most common cause of NS in children. Since the availability of steroids in 1950, glucocorticosteroids (GCs) are the first-line treatment of this disease and have radically improved the prognosis of these patients $[3,17,18,27]$. However, it is known that the long-term use of GCs affects normal growth both directly, by promoting apoptosis on osteoblasts and activating osteoclasis and indirectly, by inhibiting central growth hormone secretion $[2,10,21]$. In addition, GCs decrease bone mineralization, leading to an increased risk of fractures [11, 22, 26, 28]. This is particularly harmful for the growing skeleton, as demonstrated in children receiving long-term GC treatment for polyarthritis or inflammatory bowel disease $[14,15,28]$.

For most children with idiopathic NS, GC treatment is only intermittent with prolonged treatment-free periods during remissions and, thus, there is a need to specifically evaluate the side effects in this population. Growth delay and a deleterious effect on bone have been mainly described for patients with steroiddependent NS (SDNS) [7, 26]. We performed a study to assess both growth and bone mineral density (BMD) in the same cohort of NS patients and to compare the effects of GCs on the different subgroups according to steroid response, i.e., SDNS, steroid-sensitive NS (SSNS), and steroid-resistant NS (SRNS). We then evaluated the effect of increased ranges of GC cumulative doses with the intention to define the cutoff dose of GCs above which growth and BMD are clinically affected.

\section{Methods}

We identified retrospectively all children diagnosed with NS and followed at the University of Geneva Children's Hospital from 1992 to 2011. Patients were excluded from the analysis if they had poor therapeutic observance or were lost to follow-up. Data recorded were age at onset, gender, weight, body mass index (BMI), spine BMD and Z-score, type of NS according to GC susceptibility, number of relapses, and use of adjuvant treatment. The cumulative dose of GCs received was precisely quantified for each child during the entire observational period and reported in milligram per kilogram per day. At our institution, we prescribed only prednisone. Initial treatment for NS started at $2 \mathrm{mg} / \mathrm{kg} /$ day of GCs during 6 weeks, followed by decreased dosage on alternate days for 4 to 6 months. In the case of relapse, GC treatment was reintroduced at $2 \mathrm{mg} / \mathrm{kg} /$ day until 5 days without proteinuria, followed by alternate days of decreased dosage.
Growth evaluation

Initial height $(\mathrm{cm})$ assessed at the time of diagnosis and final height assessed at the end of the follow-up period were both reported in the $Z$-score (standard deviation [SD]) using growth curves based on the World Health Organization Child Growth Standards [29]. Growth during the follow-up period was defined as the difference $(\Delta)$ between the final and initial height, expressed as a $Z$-score.

Measurement of bone variables

Children who had at least two L2-L4 spine BMD measurements during follow-up were included in the analysis of GCs on bone. BMD was assessed by dual X-ray absorptiometry (DXA), which is recognized as the gold standard to evaluate bone mineral content and density in children and adults [3]. Examinations were performed using Hologic ${ }^{\circledR}$ (Bedford, USA) devices (QDR 2000, SN/2141 and QDR 4500, SN/ 45278) that had been cross-calibrated, thus enabling the results to be comparable over time. Spine $Z$-score was used as it is the most representative in children, according to the literature and international recommendations [4, 30].

\section{Statistical analyses}

Standard descriptive statistics were used, such as proportions and percentages, mean and SD, and median and interquartile range (IQR), depending on whether the distribution of variables was normal or not. The cumulative dose of GCs was logtransformed to obtain a normal distribution. All patients were included in the analysis of the impact of GCs on growth. Growth was defined by the difference of the height $Z$-score between baseline and final assessment ( $\Delta$ height $Z$-score). Patients were included in the analysis of the impact of GCs on bone mass if they had at least two BMD measures. Change in bone mass was defined as the difference in the $Z$-score of spine BMD between the two measures ( $\Delta$ spine $Z$-score). Patients' initial and final BMD values were compared to standard values of healthy children available in the literature [25]; paired analyses were performed using standard values matched for age and gender. Linear regressions were used to assess the association between the cumulative dose of GCs received and growth ( $\Delta$ height $Z$-score) or BMD ( $\Delta$ spine $Z$ score) in two separate univariate models. Multivariate analyses were used for adjustment on potential confounders, such as age, BMI, duration of follow-up, or baseline height/BMD values.

Finally, we performed two different analyses of subgroups. First, we analyzed patients according to the response of their disease to steroids (SSNS, SDNS, or SRNS). We then divided all patients into three GC dose-level subgroups depending on the mean cumulative dose of GCs received: low dose 
( $<0.2 \mathrm{mg} / \mathrm{kg} /$ day), medium dose $(0.2$ to $0.4 \mathrm{mg} / \mathrm{kg} / \mathrm{day})$, and high dose $(>0.4 \mathrm{mg} / \mathrm{kg} / \mathrm{day})$. All analyses were performed with Stata $12^{\circledR}$ (StataCorp, College Station, TX, USA); all tests were two-tailed and a $P$ value $<0.05$ was considered statistically significant.

\section{Results}

\section{Patient characteristics}

Of 34 patients identified, 30 were included in the study (Table 1). Four patients were excluded from the analysis: two with poor therapeutic observance and two lost to follow-up (change of address). Onset of NS occurred at a median age of 3.7 years old (IQR 2.6-4.8); median duration of follow-up was 9.8 years (IQR 6.6-11.7). Children had between 1 and 37 relapses (median, 8.5; IQR, 4-13) and received a median dose of $0.27 \mathrm{mg} / \mathrm{kg} /$ day (IQR $0.18-0.35$ ) of GCs. One half of all children $(50 \%, 15 / 30)$ presented SSNS and one half were SRNS (7/30, $23 \%$ ) or SDNS (8/30, $27 \%)$. NS etiologies were minimal change disease $(n=8)$, focal segmental glomerulosclerosis $(n=5)$, mesangial hypertrophy $(n=$ $1)$, and membranous-proliferative disease $(n=1)$.

Patients with SSNS had a median number of 4 relapses (IQR 2; 9); SRNS, 10 (IQR 2; 23); and SDNS, 14.5 (IQR 9; 21). Cumulative doses of GCs were significantly higher in patients with SRNS (median, $0.36 \mathrm{mg} / \mathrm{kg} /$ day; IQR $0.20 ; 0.54$ ) compared to patients with SSNS (median, 0.21; IQR 0.13; 0.29; $P=$ 0.05 ) and between SDNS (median 0.32; IQR 0.25; 0.43) and SSNS $(P=0.018)$. As previously described, patients were divided into three subgroups according to the mean cumulative dose of GCs received. In the low-dose group, there were seven SSNS, one SDNS, and one SRNS; in the medium-dose group, eight SSNS, four SDNS, and three SRNS; and in the high-dose group, three SDNS and three SRNS.

Impact of glucocorticosteroids on growth

Growth ( $\Delta$ height $Z$-score) of included patients tended to be negatively associated with the cumulative dose of GCs ( $P=$ $0.14, R^{2}=7 \%$ ) by univariate linear regression analysis (Fig. 1a). This association was statistically significant $(P=$ $0.04, R^{2}=15 \%$ ) when we excluded two obese patients for whom the cumulative dose of GCs in milligram per kilogram per day was underestimated due to their weight. This association was highly significant when adjusted for height $Z$-score at baseline and duration of follow-up in a multivariate model $\left(P=0.001, R^{2}=61 \%\right)$.

When splitting the 30 patients into 2 groups according to NS type, the change in height $Z$-score between initial and final assessment was significantly more important in the subgroup of patients with SDNS or SRNS (mean change, -1.02 [SD]; $95 \% \mathrm{CI}-1.77,-0.27)$ than in patients with SSNS $(-0.03$ [SD]; $95 \%$ CI $-0.54,+0.48 ; P=0.03$ ) (Fig. 2a). Moreover, the final height $Z$-scores of the subgroup of patients with SDNS or SRNS were significantly lower (mean, -0.75 [SD]; $95 \%$ CI $-1.31,-0.19)$ than those of patients with SSNS (+0.3 [SD]; $95 \% \mathrm{CI}-0.23,+0.83 ; P<0.01)$, whereas there was no difference between these two groups at baseline $(P=0.83)$.

A comparison of patient growth according to the three GC dose-level subgroups showed that although there was no difference in the baseline height $Z$-score between groups ( $P=0.29$, Table 2 ), a higher dose of GCs was strongly associated with a lower height $Z$-score at follow-up (Fig. 2b). The

Table 1 Patients' characteristics according to disease sensibility to glucocorticosteroids treatment

\begin{tabular}{|c|c|c|c|c|c|}
\hline & & All patients & SSNS & SDNS & SRNS \\
\hline Patient & $N$ & 30 & 15 & 8 & 7 \\
\hline Gender & $N$ & $21 \mathrm{M}, 9 \mathrm{~F}$ & $12 \mathrm{M}, 3 \mathrm{~F}$ & $6 \mathrm{M}, 2 \mathrm{~F}$ & $3 \mathrm{M}, 4 \mathrm{~F}$ \\
\hline Age (at NS onset) & year & $3.7(1.5,10.3)$ & $3.8(2.0,8.3)$ & $3.8(2.8,10.3)$ & $2.2(1.5,7.2)$ \\
\hline Height (at NS onset) & $\mathrm{cm}$ & $103(83,136)$ & $100(86,136)$ & $104(92,136)$ & $94(83,133)$ \\
\hline BMI & $\mathrm{kg} / \mathrm{m}^{2}$ & $16.6(13.2,21.3)$ & $16.6(13.2,21.3)$ & $17.9(14.4,20.1)$ & $16.3(15.2,20.4)$ \\
\hline Duration of follow-up & year & $9.8(1.5,17.8)$ & $8.5(2.7,15.1)$ & $10.5(3.8,17.4)$ & $9.6(1.5,17.8)$ \\
\hline Relapse & $N$ & $8.5(1,37)$ & $4(1,17)$ & $14.5(8,28)$ & $10(1,37)$ \\
\hline $\mathrm{GC}$ dose & $\mathrm{mg} / \mathrm{kg} / \mathrm{d}$ & $0.27(0.08,0.71)$ & $0.21(0.08,0.35)$ & $0.32(0.18,0.71)$ & $0.36(0.11,0.58)$ \\
\hline \multicolumn{6}{|l|}{ Diagnosis } \\
\hline Minimal change disease & $N$ & 23 & 15 & 8 & 0 \\
\hline Focal segmental glomerulosclerosis & $N$ & 5 & 0 & 0 & 5 \\
\hline Other & $N$ & 2 & 0 & 0 & 2 \\
\hline
\end{tabular}

The median values are reported with minimum and maximum

$B M I$ body mass index, $F$ female, $M$ male, $N$ number, $N S$ nephrotic syndrome, $S D$ standard deviation, $S D N S$ steroid-dependant nephrotic syndrome, SRNS steroid-resistant nephrotic syndrome, SSNS steroid-sensitive nephrotic syndrome 

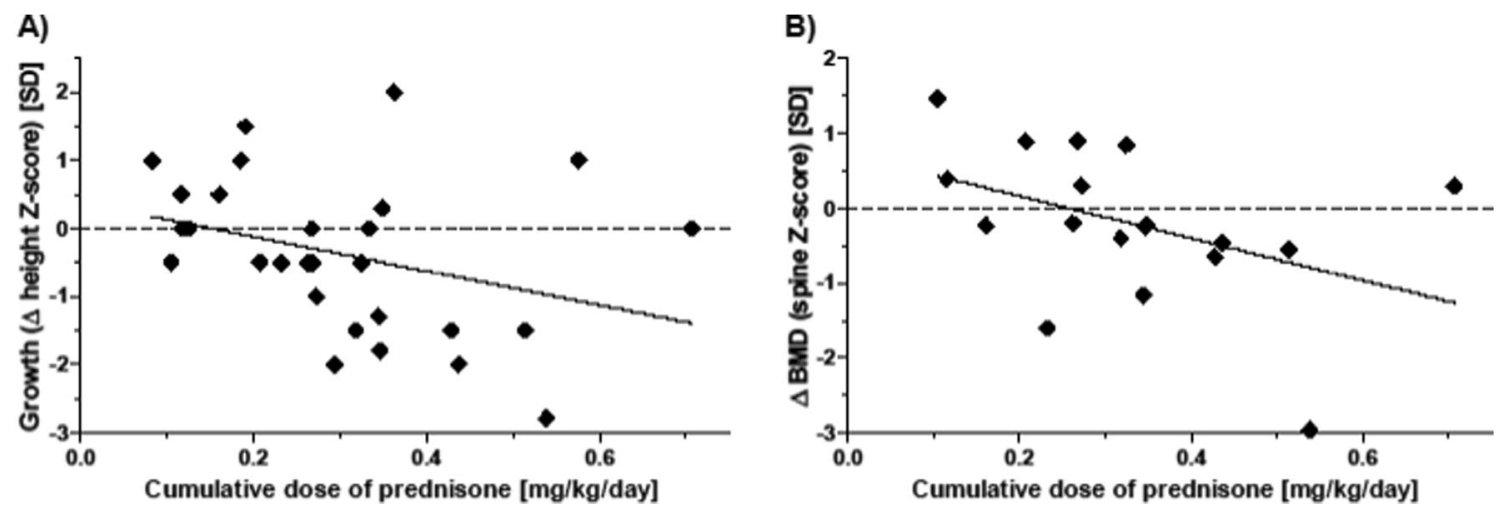

Fig. 1 Association between the cumulative dose of glucocorticosteroids and growth or bone mineral density. a Effect of cumulative dose of prednisone on growth. $\mathbf{b}$ Effect of cumulative dose of prednisone on bone mineral density

difference was statistically significant between high dose and low dose $(P<0.001)$ and high dose and medium dose $(P=$ $0.008)$ but not between low dose and medium dose $(P=0.12)$. This was confirmed by linear regression analyses, which showed an association between final height $Z$-score and the GC dose-level subgroup (using the low-dose group as the reference) in a multivariate model adjusted for confounders, such as baseline height $Z$-score and duration of follow-up (coefficient for medium-dose group -0.79 ; $95 \% \mathrm{CI}-1.44$, $-0.14 ; P=0.02$; coefficient for high-dose group $-1.58 ; 95 \%$ CI $-2.41,-0.75 ; P=0.001 ; R^{2} 61 \%$; overall $\left.P<0.001\right)$. As illustrated in Fig. 2c, the decrease in height $Z$-score per year was greater for patients in the medium-dose or high-dose groups than for those in the low-dose group in which the height $Z$-score tended to increase (Table 2).

Impact of glucocorticosteroids on BMD

Twenty patients ( 13 boys, $65 \%$; 7 girls, $35 \%$ ) had at least two DXA measurements and were included in the analyses of the impact of GCs on BMD. Seven were SSNS (35\%), 8 SDNS
(40\%), and 5 SRNS (25\%). The change in spine BMD ( $\Delta$ spine $Z$-score) was negatively associated with the cumulative dose of GCs by univariate linear regression $\left(P=0.045 ; R^{2}=\right.$ $23 \%$; Fig. 1b). In a multivariate model, this association persisted after adjustment for baseline spine BMD $Z$-score and duration of follow-up $\left(P=0.037 ; R^{2}=57 \%\right)$. A decrease in spine BMD $Z$-scores resulting in lower final BMD $Z$-scores was mainly observed in patients with SRNS or SDNS, whereas patients with SSNS were relatively spared (Table 2); however, these differences did not reach statistical significance. The difference between initial and final spine BMD Z-scores was not statistically significant in the analysis of the NS GC susceptibility and GC dose-level subgroups (Fig. 3a). Similarly, there was no difference between the subgroups in terms of change of spine BMD Z-score per year (Fig. 3b), although patients with SSNS maintained their spine BMD Z-score (median change +0.15 [SD/year]; IQR $-0.07,+0.34$ ), while patients with SRNS (median change -0.09 [SD/year]; IQR $-0.25,-0.05$ ) or SDNS (median change -0.05 [SD/year]; IQR $-0.09,+0.14)$ had a lowered spine $Z$-score.

Finally, a comparison of spine BMD values with standard $\mathrm{BMD}$ values matched for sex and age showed that that these
A)

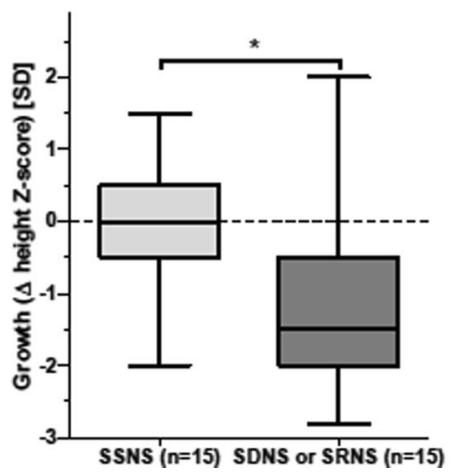

B)

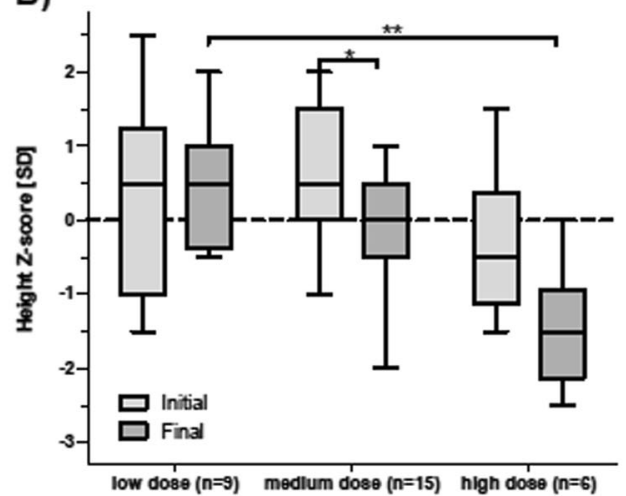

C)

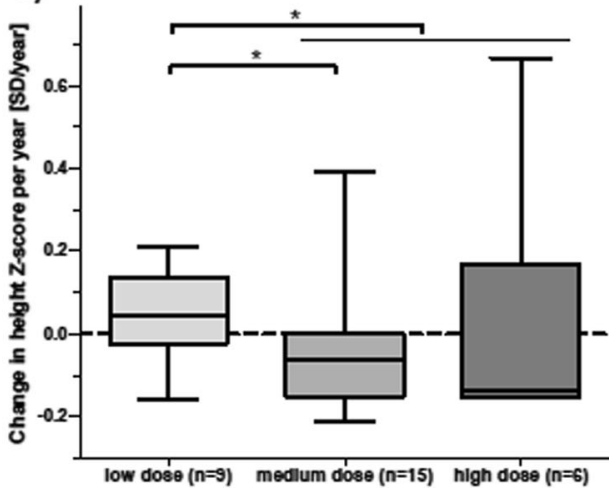

Fig. 2 Effect of glucocorticosteroids on growth in children with nephrotic syndrome. a Growth of patients with SSNS compared to those with SDNS and SRNS. b Comparison of initial and final height $Z$-score for the three GCs level subgroups. $\mathbf{c}$ Change in height $Z$-score per year for the three GCs level subgroups. $* P<0.05 ; * * P<0.01$. SDNS steroid-dependent NS, SRNS steroid-resistant NS, SSNS steroidsensitive NS 
Table 2 Subgroup analysis of the evolution of height and bone mineral density $Z$-score of patients with nephrotic syndrome

\begin{tabular}{|c|c|c|c|c|c|c|}
\hline \multicolumn{7}{|l|}{ A. NS GC susceptibility } \\
\hline Height & & All patients & SSNS $(n=15)$ & SDNS $(n=8)$ & SRNS $(n=7)$ & $P$ \\
\hline Initial height $Z$-score & SD & $0(-1.0,+1.5)$ & $0(0,+1.5)$ & $0(-1.0,+1.0)$ & $+1.0(-1.0,+1.5)$ & 0.79 \\
\hline Final height $Z$-score & SD & $-0.1(-1.3,+0.5)$ & $+0.5(-0.5,+1.0)$ & $-1.5(-1.5,-0.4)$ & $-0.3(-1.3,+0.5)$ & 0.02 \\
\hline Growth ( $\Delta Z$-score $)$ & SD & $-0.5(-1.5,+0.3)$ & $0(-0.5,+0.5)$ & $-1.0(-1.8,-0.5)$ & $-1.5(-2.5,+1)$ & 0.05 \\
\hline Change in height $Z$-score per year & $\mathrm{SD} /$ year & $-0.1(-0.2,0)$ & $0(-0.1,+0.1)$ & $-0.1(-0.1,-0.1)$ & $-0.2(-0.2,+0.4)$ & 0.10 \\
\hline Bone mineral density & & All Patients & $\operatorname{SSNS}(n=7)$ & SDNS $(n=8)$ & SRNS $(n=5)$ & $P$ \\
\hline Initial spine BMD $Z$-score & SD & $-0.3(-1.3,+0.1)$ & $-0.1(-0.3,+0.1)$ & $-0.8(-1.5,-0.3)$ & $-0.2(-1.7,+0.7)$ & 0.22 \\
\hline Final spine BMD $Z$-score & SD & $-0.3(-1.7,-0.1)$ & $-0.1(-0.3,+0.6)$ & $-0.9(-2.0,-0.2)$ & $-1.4(-1.9,-0.7)$ & 0.06 \\
\hline Change in spine $\mathrm{BMD}$ ( $\Delta Z$-score $)$ & SD & $-0.2(-0.6,+0.6)$ & $+0.3(-0.2,+0.9)$ & $-0.3(-0.6,+0.6)$ & $-0.6(-2.1,-0.3)$ & 0.35 \\
\hline Change in spine BMD $Z$-score per year & $\mathrm{SD} /$ year & $0(-0.1,+0.2)$ & $+0.2(-0.1,+0.3)$ & $0(-0.1,+0.1)$ & $-0.1(-0.3,-0.1)$ & 0.17 \\
\hline \multicolumn{7}{|l|}{ B. GC dose-level subgroups } \\
\hline Height & & All patients & Low $(n=9)$ & Medium $(n=15)$ & $\operatorname{High}(n=6)$ & $P$ \\
\hline Initial height $Z$-score & SD & $0(-1,+1.5)$ & $+0.5(-1.0,+1.0)$ & $0.5(0,+1.5)$ & $-0.5(-1.0,0)$ & 0.29 \\
\hline Final height $Z$-score & SD & $-0.1(-1.3,+0.5)$ & $+0.5(-0.3,+0.5)$ & $0(-0.5,+0.5)$ & $-1.5(-2.0,-1.3)$ & 0.01 \\
\hline Growth ( $\Delta Z$-score $)$ & SD & $-0.5(-1.5,+0.3)$ & $+0.5(0,+1.0)$ & $-0.5(-1.5,0)$ & $-1.5(-2.0,0)$ & 0.07 \\
\hline Change in height $Z$-score per year & $\mathrm{SD} /$ year & $-0.1(-0.2,0)$ & $0(0,+0.1)$ & $-0.1(-0.2,0)$ & $-0.1(-0.2,0)$ & 0.06 \\
\hline Bone mineral density & & All patients & Low $(n=4)$ & Medium $(n=11)$ & $\operatorname{High}(n=5)$ & $P$ \\
\hline Initial spine BMD $Z$-score & SD & $-0.3(-1.3,+0.1)$ & $-0.4(-1.3,0)$ & $-0.3(-1.2,+0.2)$ & $-0.4(-1.5,-0.3)$ & 0.73 \\
\hline Final spine BMD $Z$-score & SD & $-0.3(-1.7,-0.1)$ & $-0.2(-0.6,+0.6)$ & $-0.3(-1.9,0)$ & $-0.9(-2.0,-0.7)$ & 0.22 \\
\hline Change in spine $\mathrm{BMD}(\Delta Z$-score $)$ & SD & $-0.2(-0.6,+0.6)$ & $+0.8(+0.1,+1.3)$ & $-0.2(-1.2,+0.9)$ & $-0.6(-0.6,-0.5)$ & 0.06 \\
\hline Change in spine BMD $Z$-score per year & $\mathrm{SD} /$ year & $0(-0.1,+0.2)$ & $+0.2(+0.1,+0.2)$ & $-0.1(-0.2,+0.2)$ & $-0.1(-0.1,0)$ & 0.17 \\
\hline
\end{tabular}

The median values are reported with the interquartile range

$N$ number, $S D$ standard deviation, $S D N S$ steroid-dependant nephrotic syndrome, SRNS steroid-resistant nephrotic syndrome, SSNS steroid-sensitive nephrotic syndrome, $P p$ values

values were significantly lower at the end of follow-up $(P=$ 0.028; Fig. 3c).

\section{Discussion}

Although GCs are the treatment of choice for children with idiopathic NS, side effects related to both growth and bone mineralization need to be taken into account. Long-term GC treatment induces severe osteoporosis resulting in a deregulation in bone turnover $[5,7,13]$. GCs suppress bone formation by inhibiting the osteoblasts replication and differentiation and favor bone resorption by stimulating osteoclasia. In addition, in the presence of GCs, intestinal calcium adsorption by the gut and kidney is decreased $[2,5,6,10,21]$. The resulting growth delay observed in children is the result of this disequilibrium in
A)

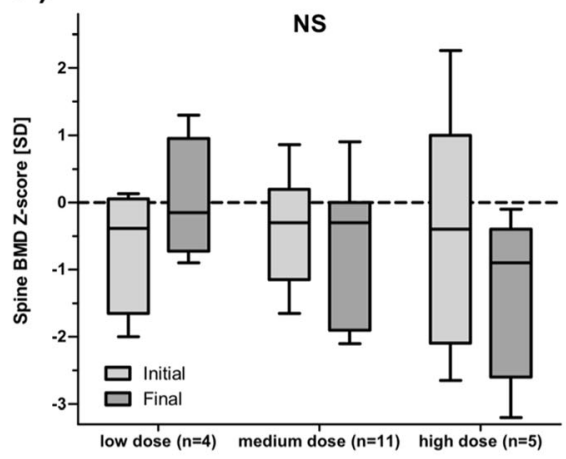

B)

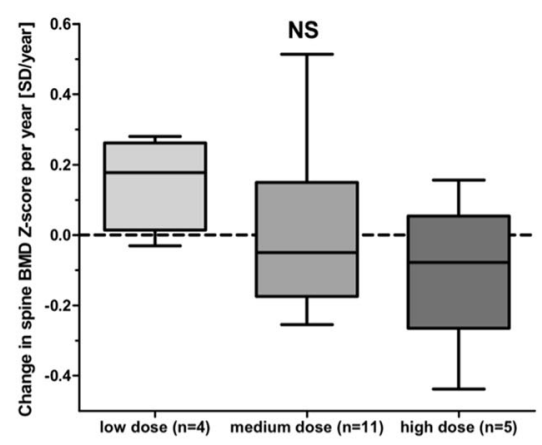

C)

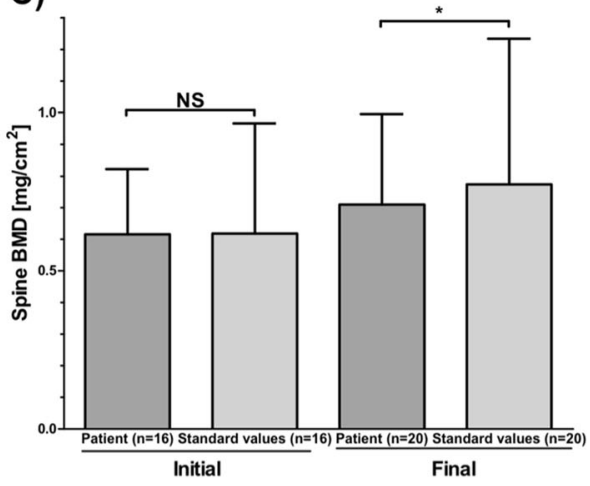

Fig. 3 Effect of glucocorticosteroids on bone mass in children with nephrotic syndrome. a Comparison of initial and final spine BMD Zscore for the three GC dose-level subgroups. b Change in spine BMD Z- score per year for the three GC dose-level subgroups. c Comparison of initial and final spine BMD of patients with nephrotic syndrome with standard BMD values. $N S$ not statistically significant; $* P<0.05$ 
bone turnover, but also indirectly by a decrease of the central released growth hormone secretion [7, 21].

In our study, the analyses clearly showed an impact of the cumulative dose of GCs on growth. Not surprisingly, patients presenting SRNS or SDNS presented more relapses and hence received higher amount of GCs, which resulted in a significant loss of height $Z$-score per year and a total loss of -1 [SD] compared to patients with SSNS receiving a lower dose. The cutoff value of the cumulative dose of GCs above which we observed a decrease in growth was $0.2 \mathrm{mg} / \mathrm{kg}$ /day and therefore concerned mainly patients with SRNS or SDNS. This value was lower than the $0.75 \mathrm{mg} / \mathrm{kg} /$ day reported by Simmond et al. in their 4.2-year analysis of children with SDNS [23].

There are differing opinions in the literature on the effect of GCs on growth, depending on NS types. Several authors agree that intermittent GC treatment, as prescribed in SSNS, has no effect on the final adult size $[7,9,12,19,24]$, which is in line with our results. This is explained by the possible periods of catch-up growth when the patient is in remission [9]. In contrast, Emma et al. report a delay of growth in patients with SDNS secondary to cumulative doses of GCs with a higher risk according to younger age at NS onset, which was not demonstrated in our population [8].

An increased risk of fracture is reported in children treated by long-term GCs for polyarthritis or inflammatory bowel disease [4]. In these particular disorders, not only the treatment but also the inflammatory state participates in the loss of bone density [15]. In children with NS who received only GCs intermittently, the risk of osteoporosis remains unclear. Previous studies have concluded that intermittent GCs had no effect on bone in children with SSNS. For example, Leonard et al. report no change in spine BMD in a cohort of 60 children with SSNS receiving intermittent treatment compared to a control group $[16,20,25]$. The same authors recently analyzed bone mineralization in 56 SSNS children by peripheral quantitative computed tomography (PQCT); trabecular and cortical PQCT were analyzed at inclusion and after 6 and 12 months' followup. GC therapy was associated with an increase in the cortical BMD Z-score, potentially related to suppress bone formation and greater secondary mineralization [25]. In our patients, the effect of GCs on the final BMD value appears to be less significant than that on growth. Even if there is a linear correlation between the cumulative dose of GCs and BMD, the differences between the GC dose-level subgroups were not significant. However, according to Fig. 1b, it appears that nearly all patients had a clinically significant decrease in BMD Z-score when treated with GC doses higher than $0.4 \mathrm{mg} / \mathrm{kg} / \mathrm{d}$. Indeed, this value is the first cutoff dose proposed in the literature and above which, bone mineralization may be impaired. Our results are in agreement with those reported by Aceto et al., who showed in 12 SSNS patients treated with GCs reduced BMD $Z$-score, obtained by DXA, after 12 months. BMD Z-score significantly correlate with the total dosage of prednisone [1].
Our study had several limitations. The first is related to the retrospective nature of this study. Some data, such as pubertal stage, bone age, and nutrition status, particularly calcium and vitamin D supplementation, are missing in the charts and were not included in the analysis. This is regrettable since these factors may also influence height and BMD values. Another limitation is the small number of patients. Our population was sufficient to confirm a dose-dependent effect of GCs on growth and BMD, but the 20 patients followed with DXA assessments may be too small to detect a significant difference between GC dose-level subgroups. Our results showed a trend that needs to be confirmed by larger studies. The strength of the study is the long follow-up, the inclusion of all types of NS (SSNS, SDNS, and SRNS), and the analysis of intermittent GCs on bone mass, which has been poorly studied.

\section{Conclusions}

Long-term GC treatment in children with NS leads to decreased growth for doses exceeding $0.2 \mathrm{mg} / \mathrm{kg} / \mathrm{day}$, and a dose above $0.4 \mathrm{mg} / \mathrm{kg} /$ day may be deleterious on skeletal development. Accordingly, it may be important to consider the use of steroid-sparing agents for children with SDNS and SRNS receiving cumulative dose of GCs above these cutoff values.

Financial disclosure The authors have no financial relationships relevant to this article to disclose.

Conflict of interest All authors declare no conflict of interest.

\section{References}

1. Aceto G, D'Addato O, Messina G et al (2014) Bone health in children and adolescents with steroid-sensitive nephrotic syndrome assessed by DXA and QUS. Pediatr Nephrol 29(11):2147-2155

2. Allen DB (1996) Growth suppression by glucocorticoid therapy. Endocrinol Metab Clin N Am 25:699-717

3. Barness LA, Moll GH, Janeway CA (1950) Nephrotic syndrome I. Natural history of the disease. Pediatrics 5:486-503

4. Bianchi M, Baim S, Bishop N et al (2010) Official positions of the International Society for Clinical Densitometry (ISCD) on DXA evaluation in children and adolescents. Pediatr Nephrol 25:37-47

5. Canalis E, Delany AM (2002) Mechanisms of glucocorticoid action in bone. Ann N Y Acad Sci 966:73-81

6. Canalis E, Mazziotti G, Giustina A et al (2007) Glucocorticoidinduced osteoporosis: pathophysiology and therapy. Osteoporos Int 18:1319-1328

7. Donatti TL, Koch VH, Fujimura MD et al (2003) Growth in steroidresponsive nephrotic syndrome: a study of 85 pediatric patients. Pediatr Nephrol 18:789-795

8. Emma F, Sesto A, Rizzoni G (2003) Long-term linear growth of children with severe steroid-responsive nephrotic syndrome. Pediatr Nephrol 18:783-788 
9. Foote KD, Brocklebank JT, Meadow SR (1985) Height attainment in children with steroid-responsive nephrotic syndrome. Lancet 2:917919

10. Friedman M, Strang LB (1966) Effect of long-term corticosteroids and corticotrophin on the growth of children. Lancet 288:569-572

11. Laan RF, van Riel PL, van de Putte LB et al (1993) Low-dose prednisone induces rapid reversible axial bone loss in patients with rheumatoid arthritis. A randomized, controlled study. Ann Intern Med 119:963-968

12. Lam CN, Arneil GC (1968) Long-term dwarfing effects of corticosteroid treatment for childhood nephrosis. Arch Dis Child 43:589594

13. Lane NE, Lukert B (1998) The science and therapy of glucocorticoidinduced bone loss. Endocrinol Metab Clin N Am 27:465-483

14. Leonard MB (2007) Glucocorticoid-induced osteoporosis in children: impact of the underlying disease. Pediatrics 119(Suppl 2): S166-S174

15. Leonard MB, Zemel BS (2002) Current concepts in pediatric bone disease. Pediatr Clin N Am 49:143-173

16. Leonard MB, Feldman HI, Shults J et al (2004) Long-term, high-dose glucocorticoids and bone mineral content in childhood glucocorticoid-sensitive nephrotic syndrome. N Engl J Med 351: $868-875$

17. Lombel RM, Gipson DS, Hodson EM (2012) Treatment of steroidsensitive nephrotic syndrome: new guidelines from KDIGO. Pediatr Nephrol 28:415-426

18. Lombel RM, Hodson EM, Gipson DS (2012) Treatment of steroidresistant nephrotic syndrome in children: new guidelines from KDIGO. Pediatr Nephrol 28:409-414

19. Madani A, Umar S, Taghaodi R et al (2011) The effect of long-term steroid therapy on linear growth of nephrotic children. Iran J Pediatr 21:21-27
20. Mishra O, Meena S, Singh S et al (2009) Bone mineral density in children with steroid-sensitive nephrotic syndrome. Indian J Pediatr 76:1237-1239

21. Mushtaq T, Ahmed S (2002) The impact of corticosteroids on growth and bone health. Arch Dis Child 87:93-96

22. Phan V, Blydt-Hansen T, Feber J et al (2014) Skeletal findings in the first 12 months following initiation of glucocorticoid therapy for pediatric nephrotic syndrome. Osteoporos Int 25(2):627-637

23. Simmonds J, Grundy N, Trompeter R et al (2010) Long-term steroid treatment and growth: a study in steroid-dependent nephrotic syndrome. Arch Dis Child 95:146-149

24. Soyka LF (1967) Treatment of the nephrotic syndrome in childhood: use of an alternate-day prednisone regimen. Am J Dis Child 113:693-701

25. Tsampalieros A, Gupta P, Denburg MR et al (2013) Glucocorticoid effects on changes in bone mineral density and cortical structure in childhood nephrotic syndrome. J Bone Miner Res 28:480-488

26. Van Staa TP, Leufkens HG, Abenhaim L et al (2000) Use of oral corticosteroids and risk of fractures. J Bone Miner Res 15:993-1000

27. van Staa TP, van Staa TP, van Staa TP et al (2002) The epidemiology of corticosteroid-induced osteoporosis: a meta-analysis. Osteoporos Int 13:777-787

28. van Staa TP, Cooper C, Leufkens $\mathrm{H}$ et al (2003) Children and the risk of fractures caused by oral corticosteroids. J Bone Miner Res 18:913-918

29. WHO Multicentre Growth Reference Study Group (2006) WHO Child Growth Standards: length/height-for-age, weight-for-age, weight-for-length, weight-for-height and body mass index-for-age: methods and development. World Health Organization, Geneva

30. Zemel BS, Kalkwarf HJ, Gilsanz V et al (2011) Revised reference curves for bone mineral content and areal bone mineral density according to age and sex for black and non-black children: results of the bone mineral density in childhood study. J Clin Endocrinol Metab 96:3160-3169 